\title{
Knowledge as a Non-equilibrium Dynamic System
}

\author{
Mikhail Oseledchik \\ Department of philosophy and social sciences \\ High school of printing and media industry \\ Moscow Polytechnic University \\ 38 Bolshaya Semenovskaya Street, Moscow, 107023, Russia \\ E-mail: balu13@yandex.ru
}

\author{
Marina Ivleva \\ Faculty of Humanities and Social Sciences \\ Department of Social Philosophy \\ Peoples' Friendship University of Russia (RUDN \\ University) \\ 6 Miklukho-Maklaya Street, Moscow, 117198, Russia \\ E-mail: marinanonna@yandex.ru
}

\author{
Vitaly Ivlev \\ National Research University \\ Moscow State Technical University named after N.E. \\ Bauman (MSTU named after N.E.Bauman) \\ 5/1 2d Baumanskaya Street, Moscow, 105005, Russia \\ E-mail: vitalijivlev@yandex.ru
}

\begin{abstract}
The article is devoted to the consideration of knowledge as a non-equilibrium, self-sustaining, and selforganizing system possessing the properties of openness, nonlinearity and dissipation, which shows the true value of learning and intellectual productive environment for possible diffusion of knowledge. The author of the article gives ten arguments in support of this point of view.
\end{abstract}

Keywords-Knowledge; non-equilibrium system; selforganization; nonlinearity; dissipation; diffusion of knowledge; attractors; intellectual productive environment; production and reconfiguration of knowledge; training; knowledge of the individual; knowledge of society

\section{INTRODUCTION}

To begin with, knowledge of the individual, being a fragment of knowledge of the community as a cultural phenomenon, and knowledge of the community as a whole are a non-equilibrium dynamic system. Here it is necessary to make a reservation that this statement is by no means a tribute to the fashionable synergetic tradition but the result of analysis of cognitive processes and their real results.

\section{KNOWLEDGE AS AN OPEN SELF-ORGANIZING SYSTEM}

Knowledge is always an open, self-organizing, heterogeneous system of exchanging information with the environment. There are several arguments for this statement.

First, history has repeatedly shown that knowledge is always a dynamic unity of heterogeneous cognitive elements, which are connected into the organic system. The change of

This paper was financially supported by the Ministry of Education and Science of the Russian Federation on the program to improve the competitiveness of Peoples' Friendship University (RUDN University) among the world's leading research and education centers in the 2016-2020 (The Agreement number 02.A03.21.0008) individual components of this system inevitably entails, at first, a change of some cluster of this system, and then the change of the entire system as a whole; ("The emergence of quantum mechanics can serve as an example of scientific revolution. Quantum theory had a significant influence not only on the natural science picture of the world, but also on the understanding of the structure of the world and objects studied by the logico-mathematical, technical and human sciences. The scope of concepts and metaphors, which are used by it, is not limited to physics") [1].

Second, a careful analysis of the content of knowledge shows that it necessarily includes rational and irrational elements, implicit and explicit knowledge, probabilistic and reliable elements, intuitive guesses and logical evidence. For example, a visual representation of the structure of a molecule that arose in Kekule or a visual idea of the structure of the atom, created by Rutherford [2. P. 203-204], which later became the basis of modern chemistry and physics, was repeatedly discussed, verified and confirmed through scientific discourse, but, fundamentally, they all were based on intuition. All these elements constantly interact with each other and are inseparably linked; moreover, "scientists reported that our brain has a built-in random number generator like roulette. It is the accident that helps us survive. For example, the antelope chased by a lion has more chances to survive by making occasional jumps in different directions. Specialists have conducted studies on cats, frogs and even jellyfish - the result is the same. The scientists concluded that uncertainty is a fundamental property which is embedded in the brain of all living beings. Random behavior, uncertainty lead to new combinations of ideas which drive the process of cognition [3. P. 25].

Third, any individual is constantly exchanging information in the communication process with other individuals and a variety of repositories and sources of codified knowledge; in this case, personal knowledge of an 
individual grows, and, at the same time, the knowledge of the community as a whole is enriched. By obtaining new knowledge, the holder and the user of this knowledge reinvents it by virtue of his personal cognitive and psychological characteristics thanks to the existing in him set of knowledge and skills. After that, he starts to produce original knowledge, often destroying the old one (for example, the telescope invented by Galileo Galilei in 1609, completely changed the mankind's concept of the Universe; having destroyed old knowledge of the Universe, a technical device which materialized the new knowledge, in this case, became a tool which destroyed the old knowledge).

Fourth, knowledge as such, being a combination of assertive (positive), negative and hypothetical components, is constantly changing at practically every moment of time, thanks to the creative activity of its carriers, users, and external influence from the practical activity of the community as a whole by acquiring new elements of knowledge by means of confirming their hypothetical components, reassessing and rejecting the assertive components which acquire the status of obsolete and erroneous. This is done by expanding the number of negative components, i.e. within the knowledge there are multidirectional processes of confirming and verifying probabilistic knowledge and constructing new positive knowledge along with refutation and destruction of old knowledge. "According to the latest research of Israeli scientists, people who drink a glass of beer, wine or a glass of some stronger beverage a day, have much more elastic arteries, which is an indicator of the health of the cardiovascular system. As Dr. Roywen Zimlichmann of the Wolfson Medical Center and Tel Aviv University says, it was previously thought that only red wine is useful, but now studies have confirmed that beer and spirits also have a positive effect on the elasticity of the arteries. With the decrease in the elasticity of the arteries, they cannot relax, which causes an increase in upper blood pressure, this fact, in its turn, is a harbinger of cardiovascular diseases, strokes, heart attacks [4. P. 25].

Fifth, it has long become clear that the knowledge of any carrier and user (individual, group, organization, community) is always systemic and holistic, and it is simultaneously included in systems of higher order (knowledge of an individual is a part of the knowledge of organization and community as a whole, instruction or a manual are a part of the knowledge of organization, etc., both at the same time are the fragments of general human knowledge and culture as a whole).

Sixth, it is obvious with the most superficial approach that knowledge is extremely unevenly distributed between carriers and users. Some have more knowledge and more effective ways of understanding, interpreting and applying this knowledge in practice than other individuals because of a variety of subjective and objective reasons of psychological, intellectual and social type [5]. This fact is the basis for the inevitability of the processes of learning, dissemination (informing) and reconfiguring knowledge in any society and its subsystems: in the process of communication, knowledge is inevitably transmitted from one individual to another or to a group of other individuals. Regardless of the awareness of these processes, of the will of the members of the society, there occur some process of cognitive diffusion, interpenetration of elements and sets of knowledge.

Quite naturally, it appear teachers, i.e. people who possess a large quantitative and qualitative knowledge, are capable of transferring this knowledge to other individuals trainees. Due to the uneven distribution of difference in their content and subject areas sets of knowledge, in the process of communication, educators and informers, on the one hand, and trainees and informants, on the other hand, quite often change their role-playing social functions and, consequently, their places in the knowledge dissemination processes without even realizing it. In other words, all individuals, depending on the context, circumstances and tasks of communication in this particular case, are both teaching and learning and informing and being informed. In this sense of knowledge, existing constantly in two hypostases as knowledge of the individual and as knowledge of the society, possesses independent activity, since any message received in the process of communication is decoded and assimilated by the subject of knowledge in whole or in part and affects the entire system of knowledge that the subject as a whole has developed.

Seventh, knowledge automatically responds to the impact of the external environment, opposing it or adapting to it through its internal reorganization (in spite of the practical and psychological importance of weather for each person, meteorology as a science did not exist prior to the beginning of the nineteenth century). "The problem partly was that, in order to obtain satisfactory results in meteorology, there are needed exact temperature measurement and thermometers for a long time that were difficult to produce. For getting accurate readings, it was necessary to make in the glass tube a very smooth hole, and it was not easy. The first person who solved this problem, was a Dutch master of instruments Gabriel Daniel Fahrenheit [6. P. 306]. In other words, knowledge of weather, in spite of the social demand for the emergence and development of meteorology as a system of knowledge about weather with inherent to it, counteracted this influence of the environment because it did not have the technological base and logistical support. Yet, as a problem of social environment was set, the material conditions were finally created and knowledge was able to adapt to the requirements of the environment at some point in time.

Eighth, the new knowledge almost always plays the role of a destabilizing factor which unbalances the system and thereby leads it to the processes of "self-organization" evolutionary changes in the system. In 1924, Edwin Hubble's article "Cepheids in Spiral Nebulae" was published, it proves that the universe consists not only of the Milky Way but of a large number of individual galaxies, many of which are larger and more remote than the Milky Way. When carrying out measurements of individual galaxies, he made the discovery that all galaxies remove from us, and their speeds are almost exactly proportional to the distances: the further the galaxy, the faster it moves. Thus, the thought of an expanding Universe arose. For a long time, this idea destabilized all cosmology and all our knowledge of the 
structure and origin of the world. But then, having analyzed the ideas of Hubble, scientists, by using the concepts of Einstein, Georges Lemaitre and other great thinkers, came to the theory of the Big Bang, which is now evolutionarily evolving and is justified, i.e. is organizing itself, becoming the fundamental postulate of modern cosmology [7. P. 306].

Ninth, knowledge has a system of accumulation, preservation, transmission and application of information (by means of its codification and recording on various media from the moment of appearing the first rudiments of writing in any of its forms. It must be noted that all the objects of material culture, apart from their immediate practical functions, have additional function of codification and preservation of knowledge, which was at the basis of the idea, the task of its production and the very process of its creation like objectifying this knowledge. If you recall the long and complicated history of the appearance of marine chronometers, you can see that this history is based, on the one hand, on the solution of purely technical problems of constructing a compact, light and at the same time very accurate device for measuring time. On the other hand, it began with the need to develop the exact foundations of navigational art, the need to create a reliable navigation system and the knowledge that it is the time which can serve as the most reliable way to determine the location of a ship in the sea. In 1510, the Spaniard Santo Cruz proposed a simple and affordable way of determining the geographical coordinates of the ship in the sea: by taking any place on Earth as a starting point (for example, now this starting point is considered to be Greenwich) and having a very accurate clock, he offered to make calculations considering time at the starting point and local time. If you multiply the difference between them by 15 , you get the exact longitude [8].

Tenth, knowledge evolves, complicating its structural and functional organization. In this process, bifurcations are possible, which leads to unpredictability of the way and methods of developing knowledge in all the diversity of its forms and historical states (for example, the discovery of $\mathrm{x}$ rays which turned the course of development of both natural, and technical sciences, and which produced a kind of revolution in medicine).

\section{KNOWLEDGE AS A NON-EQUILIBRIUM SYSTEM}

In other words, we can state that an open system of knowledge cannot be equilibrium because its functioning requires the continuous receipt of new information from the external environment, as a result of which the imbalance in the system increases. Consequently, the former relationship between the elements of the system, that is, its previous structure, is destroyed; new interconnections between the elements arise and the number of new elements sharply increases. Thus, within this system irreversible processes of destruction and loss of knowledge occur, plus reconfiguration and increment of knowledge.

Such a system is always characterized by the process of self-organization. The evolution of complex non-equilibrium systems is considered as a process of self-organization in them. Self-organization means the formation in the system of a certain ordered structure without external organizing influence. This process is nonlinear, the development of knowledge is ambiguous, multivariate; the pace of its development is constantly changing.

The term "self-organizing system" was introduced by cyberneticist William Ross Ashby to describe cybernetic systems. Self-organizing systems are characterized by:

- the ability to actively interact with the environment, change it in the direction that ensures a more successful functioning of the system;

- the presence of a certain flexibility of the structure or adaptive mechanism developed in the course of evolution;

- unpredictability of the behavior of self-organizing systems;

- the ability to take into account past experience or the possibility of learning.

Knowledge as a self-organizing system has all its basic properties, such as:

- openness;

- nonlinearity;

- dissipativity - the state of dispersion of incoming information.

Open systems are such systems that are maintained in a certain state due to continuous inflow from outside and (or) outflow of matter, energy or information. The inflow and outflow are usually of a voluminous nature, i.e. they occur at each point of the system. All knowledge holders have a constant exchange of information with other holders, this being accompanied by constant revision, criticism and reconfiguration of information. Knowledge is in the process of constant dynamic change. The constant inflow and outflow of information is a necessary condition for the existence of non-equilibrium, unstable states, in contrast to closed systems which inevitably tend to a homogeneous equilibrium state (in accordance with the second law of thermodynamics). One of the typical examples of such nonequilibrium is the so-called digital split - a situation where a part of society is mastering digital technologies, and another part may even not suspect of their existence. The nonequilibrium and instability of open systems are generated by the constant struggle of two tendencies. The first is the generation and strengthening of heterogeneities, structuring, and localization of the elements of an open system. The second is the scattering of heterogeneities, their "erosion", diffusion, and deconstruction of the system. If the first tendency wins, the open system becomes a self-organizing system, and, if the second dominates, the open system dissipates, becoming chaos. When these tendencies are approximately equal to each other in the open systems, a key role can be played by random factors and fluctuation processes along with the regular and necessary factors. Sometimes, the fluctuation can become so strong that the existing organization is destroyed. Open systems are irreversible systems; the time factor is important in them [9]. 
Non-equilibrium systems, through selectivity to external influences of the environment, perceive differences in the external environment and" take them into account "in their functioning. However, some weak actions may have a greater impact on the evolution of the system than those ones which, although being stronger, are not adequate to their own tendencies of the system. In other words, the superposition principle does not apply to nonlinear systems: here are possible the situations where the effect of the combined impact of causes A and B has nothing to do with the results of the impacts of A and B taken separately. The processes in nonlinear systems often have a threshold character. With a smooth change in external conditions, the behavior of the system changes abruptly. In other words, in states that are far from equilibrium, very weak perturbations can be amplified to gigantic waves which destroy the existing structure and contribute to its radical qualitative change. For each system, there is an optimal "corridor of non-linearity", which contributes to the formation of structures. A very strong nonlinearity, as well as a very weak nonlinearity, is incompatible with the formation of local structures. But within the limits of only the optimal "corridor", the strengthening of nonlinearity increases the number of methods of creating and forms of local structures, as well as the number of variants of the evolution of the system, its routes to the future. Nonlinear systems, being nonequilibrium and open, create and maintain themselves the heterogeneities in the environment. In such circumstances between the system and the environment, there can sometimes be created an inverse positive relationship, i.e. the system influences its environment in such a way that in the environment can be created conditions which in their turn cause changes in the system itself. For example, during the chemical reaction, there is an enzyme whose presence stimulates the production of itself is produced. The consequences of this kind of interaction of the open system and its environment can be the most unexpected and unusual [10].

An example of such nonlinearity can be considered the appearance of formalized languages in science (primarily in mathematics and logic) and the ideas of atomism, which radically changed all the views of the world, the emergence of quantum-relativistic physics, and so on [11]. Another example is the emergence of digital technologies which fundamentally transformed knowledge about the world, technology and the opportunities for activity, including purely scientific one [12].

All these features characterize knowledge in general. Using the terminology and ideology of I.Prigozhin and S.N. Kurdyumov, we can regard knowledge as a dissipative system, in which entropy constantly increases. In fact, knowledge as a whole is a unity of knowledge of an individual and knowledge of the society, which has a codified core and a huge, essentially non-codified peripheral content, defined by the intellectual, psychological and everyday circumstances of an individual's being.

The distribution of knowledge between individuals is completely uneven and asymmetrical, as well as the principal opportunities for the growth of individual knowledge.
Consequently, the intensity of development of knowledge of specific individuals and their various social groups is fundamentally different [13].

The assimilation of general knowledge by weaker individuals and marginal social groups leads to its disorganization, since these bearers of knowledge are characterized by ignorance and fragmentary perception of cognitive units [14].

On the other hand, stronger individuals and their communities face a constant increase in the flow of information that blurs the established orderly structures of the organization of knowledge. Nevertheless, the general knowledge system is able to digest and organize the received chaotic avalanches of new knowledge according to the structures of attractors which exist within the knowledge system. These are sets of the most stable formations and forms to which the processes in dissipative environments evolve. In particular, such structures-attractors include traditional forms of development of knowledge such as a problem, a hypothesis and a theory in the form of which new knowledge is fixed.

\section{CONCLUSION}

Consequently, knowledge as a whole can be viewed as a self-sustaining, self-organizing structure. As S.N. Kurdyumov pointed out, an orderly structure-process grows out of chaos, and chaos, in its turn, takes the process out to one of the structure-attractors: it generates order [15. P. 75].

This non-equilibrium of the knowledge system and its uneven distribution between carriers and users (by carriers and users we mean individuals, communities, organizations and society as a whole), explains the need for learning as a process of flowing from a more erudite carrier and knowledge user to the less erudite one, from the "teacher" to the "student", and for organizing the life of the cognizing subject within the framework of an intellectual productive environment that creates the possibility of diffusion of knowledge. It shows the significance in the system of developing the knowledge of individuals who are capable of creative production and reconfiguration of knowledge, and it allows us to use the concept of "scale-free networks" for analyzing and developing the concept of "knowledge" and a fundamentally new methodology for implementing knowledge management.

\section{REFERENCES}

[1] Kryukova I.G. Typology of scientific revolutions [Electronic resource] I I.G.Kryukova. - Access mode: http://science. logistics gr.com/index.php?option $=$ com_content\&view $=$ article\&id $=$ 1099:2012-02-28-06-55-03\& catid $=51: 2011-11-08-20-34-14 \&$ Itemid $=55$. 09.07.2014.

[2] Knyazeva E.N., Kurdyumov S.N. The foundations of synergetics: a man constructing himself and his future [Text] / E.N. Knyazeva. Moscow: KomKniga, 2010. - 232 p.

[3] Our brain plays roulette [Text] / Man and Science.- June 2002. - P. 25.

[4] Ibid. - P. 25

[5] Chistyakov D. Social Dimension of Media Space in the Age of Postmodernity. In the Context of Objective Knowledge Obtainment // 
Proceedings of the 2016 International Conference on Arts, Design and Contemporary Education. (ICADCE 2016). Advances in Social Science, Education and Humanities Research. Paris: Atlantis Press, 2016, v. 64, pp. 297-300.

[6] Bryson, B. A Brief history of almost everything in the world [Text] / Bill Bryson; Trans. from English. V. Mikhailov. - M .: AST, 2013. $607 \mathrm{p}$.

[7] Ibid.

[8] [Electronic resource]. Access mode: http://www.popmech.ru/technologies/8377-gps-Xviii-veka-morskiekhronometry/\#full. - 11.07.2014.

[9] [Electronic resource]. Access mode: http://sbiblio.com/biblio/archive/naydishev_koncepcija/15.aspx. 19.08.2012.

[10] Ibid.

[11] Inozemtsev V.A., Ivlev V.V., Ivleva M.L., Oseledchik M.B. Representology in the System of Modern Epistemology //Proceedings of the 2016 3rd International Conference on Education, Language, Art and Inter-cultural Communication (ICELAIC 2016). Advances in Social Science, Education and Humanities Research. Paris: Atlantis Press, 2017. Volume 40. P. 697-701.

[12] Oseledchik M.B., Inozemtsev V.A., Ivlev V.Y., Ivleva M.L. Logical-Philosophical Approach to the Interpretation of the Concept of Knowledge //Proceedings of the 2016 3rd International Conference on Education, Language, Art and Inter-cultural Communication (ICELAIC 2016). Advances in Social Science, Education and Humanities Research. Paris: Atlantis Press, 2017. Volume 40. P.297301.

[13] Chistyakova Olga V. Philosophical-Anthropological Meanings of Postmodernism: "Mediatizing" Human // Proceedings of the International Conference on Contemporary Education, Social Sciences and Humanities. Advances in Social Science, Education and Humanities Research (ICCESSH 2016). Volume 74. ISBN (on-line): 978-94-6252-215-2. Paris: Atlantis Press, 2016. P. 637-642.

[14] Chistyakova, Olga V. "Relationship of Self and Other in Cultural and Religious Communications // Proceedings of the International Conference on Education, Language, Art and Inter-Cultural Communication. (ICELAIC 2016). Advances in Social Science, Education and Humanities Research, Volume 40. Paris: Atlantis Press, 2017. ISBN (on-line): 978-94-6252-292-3. P. 660-664.]

[15] Knyazeva E.N., Kurdyumov S.N. The foundations of synergetics: a man constructing himself and his future [Text] / E.N. Knyazeva. Moscow: KomKniga, 2010. - 232 p. 